\title{
The value of dehydroepiandrosterone sulfate measurement in the differentiation between benign and malignant adrenal masses
}

Massimo Terzolo, Anna Alì, Giangiacomo Osella, Giuseppe Reimondo, Anna Pia, Paola Peretti, Piero Paccotti and Alberto Angeli

Dipartimento di Scienze Cliniche e Biologiche, Medicina Interna, Azienda Ospedaliera San Luigi, Università di Torino, Italy

(Correspondence should be addressed to M Terzolo, Clinica Medica, A. O. San Luigi, Regione Gonzole, 10, 10043 Orbassano (TO), Italy; Email: terzolo@usa.net)

\begin{abstract}
Objective: Owing to their increasing rate of discovery as incidental findings, the characterization of adrenal masses is an important diagnostic problem which frequently challenges the clinician's skill. Design: The results of dehydroepiandrosterone sulfate (DHEAS) measurement were evaluated in a consecutive series of 107 patients with an adrenal mass (39 men, 68 women aged 15-81 years, median 56 years). DHEAS levels observed in the patients were categorized as reduced, normal or elevated according to sex- and age-adjusted reference ranges obtained by measuring DHEAS in 214 healthy women and 142 healthy men aged 17-93 years. The working hypothesis was that a low DHEAS level is a marker of benignity.

Methods: In 84 patients, the adrenal mass was discovered serendipitously, while in the remainder the mass was clinically symptomatic. Patients with known extra-adrenal malignancies were excluded. The adrenal masses were categorized as benign or malignant by computerized tomography (CT) criteria. All patients with suspected malignant tumors or with overtly hypersecreting tumors underwent adrenalectomy. The patients with a presumptive benign tumor were followed-up for at least 12 months.

Results: In the overall series, the sensitivity and specificity of a low DHEAS level in the identification of a benign lesion were $41 \%$ and $100 \%$ respectively. Superimposable figures were obtained when considering only adrenal incidentalomas. DHEAS levels in adrenal cancers displayed some overlap with adrenal adenomas, but in only 2/11 adrenal cancers were DHEAS levels normal, while they were elevated in the remainder.

Conclusions: The present data suggest that DHEAS measurement may help to differentiate benign from malignant adrenal masses as a complementary test of CT in a clinical research setting. The value of DHEAS measurement in general practice is limited because it may be difficult to differentiate between low and low-normal values, particularly in the elderly.
\end{abstract}

European Journal of Endocrinology 142 611-617

\section{Introduction}

Characterization of adrenal masses is an important diagnostic problem which challenges the clinician's skill with increasing frequency. An adrenal mass is often found on abdominal ultrasonography or computerized tomography (CT) scans but the discovery is unexpected and unrelated with the indication for the imaging study in the majority of the patients $(1,2)$. These incidentally discovered adrenal masses, or adrenal incidentalomas, represent a classical example of a 'disease of advanced technology' and are currently being diagnosed in millions of people worldwide $(3,4)$. For these reasons, many clinical investigations have addressed the diagnostic approach to adrenal incidentalomas (4-6). A number of strategies have been recommended for hormonal screening and radiological imaging but the most critical issue in current practice, i.e. the differentiation between benign and malignant lesions, is still surrounded by controversy and uncertainty.

Low dehydroepiandrosterone sulfate (DHEAS) levels are frequently observed in patients with adrenal incidentalomas even if the reason for this reduction remains to be fully clarified (7-10). Conversely, high DHEAS levels are a marker of adrenal androgen excess that may herald the presence of an adrenal carcinoma (11). 
The aim of the present study was to test the efficacy of DHEAS measurement to characterize an adrenal mass as benign or malignant in a series of patients referred to our center for the evaluation of an adrenal mass, either of incidental discovery or clinically overt.

\section{Subjects and methods}

Cases were drawn from a series of patients with an adrenal mass referred to our center from 1990 to 1996. Patients with known extra-adrenal malignancies or patients with pre-treated adrenal cancers were excluded from the study. The 107 patients $(39$ men and 68 women, aged 15-61 years, median age 58 years) were hospitalized and followed by the same physicians (MT, A A and GO). The patients volunteered for the study and gave their informed consent. Clinical and endocrine data of 25 patients have previously been reported but the results of DHEAS measurement were analyzed with a different endpoint (7). In that report, DHEAS levels were, indeed, considered as a marker of cortisol hypersecretion by the adrenal incidentaloma.

In 84 patients, the adrenal mass was discovered serendipitously with diagnostic procedures performed for extra-adrenal complaints. These patients did not show any signs or symptoms suggestive of adrenal hypersecretion. In the other 23 patients, the adrenal mass was clinically apparent because of hormone secretion, or mass effect, or both.

All patients were studied with an abdominal CT scan and an endocrine work-up aimed to define the diagnosis. Patients with adrenal incidentaloma underwent a specific endocrine study including measurement of adrenocorticotropin (ACTH) and cortisol at $0800 \mathrm{~h}$ and $2400 \mathrm{~h}, 24-\mathrm{h}$ excretion of urinary free cortisol (UFC), plasma renin activity (PRA) and aldosterone in orthostatic posture, 24-h excretion of urinary catecholamines and vanillylmandelic acid, and suppression of cortisol after $1 \mathrm{mg}$ dexamethasone, as previously described (12). An attenuation value of 10 or less Hounsfield Units on unenhanced CT scan, and 30 or less Hounsfield Units on enhanced CT scan were considered as suggestive of a benign adrenal adenoma $(13,14)$. Other CT features considered as suggestive of a benign lesion were size less than $4.0 \mathrm{~cm}$, round or oval shape, homogeneous content and well-defined margins (5). CT scans were reviewed by an experienced radiologist. After a thorough evaluation of CT pictures, a presumptive diagnosis of benign tumor (adrenocortical adenoma, cyst, myelolipoma) was formulated when all the radiological criteria of a benign mass were satisfied, the so called 'benign CT pattern'. Conversely, when CT features of the mass were large size, non homogeneous content with irregular shape and margins, possibly with infiltration of surrounding tissues or distant metastases, or both, a 'malignant CT pattern' was assumed. When only some of the radiological criteria of benignity were present (e.g. small mass with focal heterogeneous spots or with borderline attenuation values) an 'inconclusive CT pattern' was assumed.

The patients whose adrenal incidentalomas displayed a benign CT pattern (presumed cortical adenoma) were followed-up for at least 12 months with re-evaluation of physical examination, CT scan and hormone measurements. Adrenal incidentalomas featuring inconclusive or malignant CT patterns were removed.

Pathological specimens were reviewed by an experienced pathologist. Histological diagnosis of adrenocortical carcinoma was based mainly upon the presence of regressive changes (i.e. necrosis, hemorrhage, fibrosis or calcification), invasion of the adrenal capsule, blood vessel wall, or both, and atypical mitotic figures (15).

For patients presenting with signs of overt hormone hypersecretion, the diagnosis of adrenal Cushing's syndrome rested on the clinical picture, demonstration of elevated UFC, elevated serum cortisol with disturbance of the 24-h rhythm, inadequate cortisol suppression after 1 and $8 \mathrm{mg}$ dexamethasone, and CT findings. The diagnosis of aldosterone-producing adenoma rested on the demonstration of high plasma aldosterone to PRA ratio $(>50)$, unsuppressed aldosterone after Florinef, and a CT picture consistent with an adrenal adenoma in a hypertensive patient. The diagnosis of pheochromocytoma rested on the demonstration of elevated urinary catecholamines and high vanillylmandelic acid, and on CT imaging. The hypersecreting tumors were removed, with confirmation of the diagnosis and normalization of the previously elevated hormone parameters.

Measurement of DHEAS levels was performed at $0800 \mathrm{~h}$ in all the patients and also in 214 healthy women and 142 healthy men aged 17-93 years, to define normal ranges as a function of gender and age, considering the 3rd and the 97th percentile in the agegroups $<40$ years, between 40 and 60 years and $>60$ years for each gender. DHEAS levels observed in the patients were categorized as reduced, normal or elevated according to these sex- and age-adjusted reference ranges. The standard score (z-score) of DHEAS was calculated for each patient according to the formula: standard score $(\mathrm{z})=($ raw score-mean $) / \mathrm{s}$.D., where 'raw score' was the DHEAS value of the patient under examination and 'mean' and 'S.D.' were, respectively, the mean and the standard deviation of DHEAS obtained in the subgroups of sex- and age-matched controls. Control subjects included fellows of the university, members of medical staff and their siblings, and outpatients referred for euthyroid sporadic goiter or benign prostatic hypertropy, or women referred for postmenopausal osteoporosis screening. In all the subjects, other relevant diseases were excluded by physical examination, standard biochemical and radiological screening and they were off medication. They volunteered for the study and gave their informed consent.

Hormonal variables were measured by high pressure 
liquid chromatography, RIA or immunoradiometric assay methods, using commercially available kits. All hormone assays were performed in the same laboratory. Intra- and interassay coefficients of variation for all the above mentioned variables were less than $8 \%$ and $12 \%$ respectively. In particular, serum DHEAS was determined by a RIA kit purchased from Diagnostic Systems Laboratories (Webster, TX, USA) with intra- and interassay coefficients of variation of $7.8 \%$ and $10.2 \%$ respectively. The sensitivity of the assay was $0.14 \mu \mathrm{mol} / \mathrm{l}$ $(5 \mu \mathrm{g} / \mathrm{dl})$ and the highest limit of the standard curve was $21.7 \mu \mathrm{mol} / \mathrm{l}(800 \mu \mathrm{g} / \mathrm{dl})$.

All data were analyzed with a software program (Statistica, Microsoft Corp., Tucla, OK, US) run on a personal computer. Statistical analysis was performed using two-tailed parametric or non-parametric methods for unpaired data, as appropriate. Single and multiple regression analyses were carried out to determine, first, which variables may influence DHEAS levels and, secondly, the relationship of the occurrence of a benign lesion as a function of age, gender, CT size of the mass, and hormone data. Data are expressed as the median and range. Levels of statistical significance were set at $P<0.05$.

In order to evaluate the diagnostic value of DHEAS measurement, the standard methods for calculating sensitivity and specificity were used; sensitivity $=$ true positive/(true positive + false negative); specificity $=$ true negative/(true negative + false positive). The working hypothesis was that low DHEAS is a marker of benignity. In relation to this assumption, a benign lesion with low DHEAS qualified as a true positive, a malignancy with normal or elevated DHEAS as a true negative, a benign lesion with normal or elevated DHEAS as a false negative, and a malignancy with low DHEAS as a false positive.

\section{Results}

The categorization of CT patterns of the 107 adrenal tumors is given in Table 1 .

In the 84 patients with an incidentally discovered adrenal mass, indications for surgery were: (a) inconclusive or malignant CT patterns; (b) demonstration of catecholamine excess or primary aldosteronism. Surgery was not recommended in some patients but they nevertheless chose this option. During the follow-up of the patients with a presumed benign adrenal adenoma, no signs of overt hypersecretion or of extra-adrenal malignancy became manifest. The size of the mass was found to be increased by more than $1 \mathrm{~cm}$ at the CT re-evaluation in only 2 patients with an otherwise benign CT pattern who underwent adrenalectomy. Pertinent clinical information and histological diagnoses of the patients with an adrenal incidentaloma who underwent adrenalectomy are given in Table 2 .

In 23 patients the mass had overt clinical manifestations. Full-blown Cushing's syndrome was observed in 10 patients and was associated with an adrenal adenoma
Table 1 CT patterns of the adrenal masses.

\begin{tabular}{lcc}
\hline & Incidental masses & Clinically overt masses \\
\hline Benign & $70 / 84(83 \%)$ & $13 / 23(56 \%)$ \\
Inconclusive & $12 / 84(14 \%)$ & - \\
Malignant & $2 / 84(3 \%)$ & $10 / 23(44 \%)$ \\
\hline
\end{tabular}

in 6 cases and with an adrenocortical carcinoma in the remainder. Five other adrenal carcinomas were found and removed because of symptoms related to androgen excess or mass effect. All patients with a histologically proven adrenal cancer had a local recurrence after radical surgery or a disease progression after surgical debulking in the follow-up period. Five aldosterone producing adenomas, two pheochromocytomas and one hemorrhagic cyst were also removed. Pertinent clinical information and histological diagnoses of the patients with a clinically overt adrenal tumor who underwent adrenalectomy are given in Table 3.

The concordance between CT and histological diagnoses was fairly good in the masses with malignant CT patterns $(92 \%)$ while $89 \%$ of the masses with inconclusive CT patterns were benign at histology.

The median size of the incidental masses was $3.0 \mathrm{~cm}$ (range 1.0-15) while it was $4.0 \mathrm{~cm}(1.0-13)$ for the clinically overt masses $(P=0.009)$. In patients with presumed benign masses, DHEAS was normal in 59\% of cases and reduced in $41 \%$; none of the benign masses showed high DHEAS levels. In patients with adrenal malignancies, DHEAS was normal in $25 \%$ of cases and elevated in 75\%; none of the malignant masses showed low DHEAS levels.

DHEAS levels observed in the patients with adrenal malignancies (11 primary adrenal carcinomas and one metastasis of a previously unknown lung cancer) were significantly higher than those recorded in patients with benign masses (median, range) $(17.7 \mu \mathrm{mol} / \mathrm{l}, 0.7-54.3$ $(655 \mu \mathrm{g} / \mathrm{dl}, 27-2000)$ vs $1.4 \mu \mathrm{mol} / \mathrm{l}, 0.1-8.8(53 \mu \mathrm{g} / \mathrm{dl}$, 5-324), $P<0.001$ ) (Fig. 1). The difference between malignant and benign tumors is confirmed by analyzing DHEAS levels as z-score $(7.04 \pm 7.2$ vs $-0.98 \pm 0.7$; $P<0.0001)$

The age difference between these two groups was at the limit of statistical significance (47 years $(19-65)$ for adrenal malignancies vs 56 years (15-81) for benign masses, $P=0.048)$ while gender distribution was not significantly different. The comparison between histologically proven cortical adenomas and cortical carcinomas yielded a highly significant difference $(0.9 \mu \mathrm{mol} / \mathrm{l}, 0.1-8.8$ (34 $\mu \mathrm{g} / \mathrm{dl}, 5-324)$ vs $18.4 \mu \mathrm{mol} / \mathrm{l}$, $2.8-54.3(680 \mu \mathrm{g} / \mathrm{dl}, 105-2000), P<0.001)$.

In designing a multivariate model to consider which variables might influence DHEAS levels in the present series, we selected those which, from the table of correlation values, appeared most likely to be related. The model that accounted for the biggest proportion of variance $\left(r^{2}\right)$ in 
Table 2 Pertinent clinical data and histological diagnoses of the patients with adrenal incidentalomas who underwent surgery.

\begin{tabular}{|c|c|c|c|c|c|c|c|}
\hline Patient & Sex & $\begin{array}{c}\text { Age } \\
\text { (years) }\end{array}$ & $\begin{array}{l}\text { CT size } \\
(\mathrm{cm})\end{array}$ & Reason for surgery & Histology & $\begin{array}{c}\text { DHEAS } \\
(\mu \mathrm{g} / \mathrm{dl})-(\mu \mathrm{mol} / \mathrm{l})\end{array}$ & Stage \\
\hline 1 & $\mathrm{~F}$ & 61 & 5 & Size, high catecholamines & Pheochromocytoma & $10-0.2$ & - \\
\hline 2 & $\mathrm{~F}$ & 81 & 6 & $\begin{array}{l}\text { Inconclusive CT pattern } \\
\text { (size, focal heterogeneity) }\end{array}$ & Myelolipoma & $39-1.0$ & - \\
\hline 3 & $\mathrm{~F}$ & 60 & 7 & $\begin{array}{l}\text { Inconclusive CT pattern } \\
\text { (size) }\end{array}$ & Myelolipoma & $20-0.5$ & - \\
\hline 4 & $\mathrm{~F}$ & 71 & 3 & $\begin{array}{l}\text { Inconclusive CT pattern } \\
\text { (heterogeneous mass) }\end{array}$ & Pheochromocytoma & $10-0.2$ & - \\
\hline 5 & $\mathrm{~F}$ & 64 & 3.5 & Increased at follow-up & Cortical adenoma & $7-0.18$ & - \\
\hline 6 & $\mathrm{~F}$ & 69 & 3.5 & $\begin{array}{l}\text { Inconclusive CT pattern } \\
\text { (borderline attenuation) }\end{array}$ & Cortical adenoma & $30-0.8$ & - \\
\hline 7 & $\mathrm{~F}$ & 52 & 4.2 & $\begin{array}{l}\text { Inconclusive CT pattern } \\
\text { (size, focal heterogeneity) }\end{array}$ & Cortical adenoma & $5-0.1$ & - \\
\hline 8 & $M$ & 46 & 3 & $\begin{array}{l}\text { Inconclusive CT pattern } \\
\text { (irregular margins) }\end{array}$ & Cortical adenoma & $16-0.4$ & - \\
\hline 9 & $\mathrm{~F}$ & 49 & 2.5 & Patient willingness & Cortical adenoma & $98-2.6$ & - \\
\hline 10 & $\mathrm{~F}$ & 60 & 5.5 & $\begin{array}{l}\text { Inconclusive CT pattern } \\
\text { (size, focal heterogeneity) }\end{array}$ & Cortical adenoma & $100-2.7$ & - \\
\hline 11 & M & 61 & 2 & Patient willingness & Cortical adenoma & $102-2.7$ & - \\
\hline 12 & M & 47 & 3 & $\begin{array}{l}\text { Inconclusive CT pattern } \\
\text { (heterogeneous mass) }\end{array}$ & Hemorrhagic cyst & $129-3.5$ & - \\
\hline 13 & M & 66 & 7 & Size, high catecholamines & Pheochromocytoma & $167-4.5$ & - \\
\hline 14 & $\mathrm{~F}$ & 55 & 4 & Increased at follow-up & Cortical adenoma & $16-0.4$ & - \\
\hline 15 & M & 65 & 3 & $\begin{array}{l}\text { Inconclusive CT pattern } \\
\text { (irregular margins) }\end{array}$ & Cortical adenoma & $60-1.6$ & - \\
\hline 16 & $\mathrm{~F}$ & 42 & 3.7 & $\begin{array}{l}\text { Inconclusive CT pattern } \\
\text { (heterogeneous mass) }\end{array}$ & Cyst & $218-5.9$ & - \\
\hline 17 & $\mathrm{~F}$ & 59 & 4 & $\begin{array}{l}\text { Inconclusive CT pattern } \\
\text { (size, focal heterogeneity) }\end{array}$ & Cortical adenoma & $53-1.4$ & - \\
\hline 18 & $\mathrm{~F}$ & 19 & 13 & Malignant CT pattern & Adrenocortical cancer & $1000-27$ & II \\
\hline 19 & $\mathrm{~F}$ & 45 & 15 & Malignant CT pattern & Adrenocortical cancer & $384-10.4$ & II \\
\hline 20 & $\mathrm{~F}$ & 65 & 3 & $\begin{array}{l}\text { Inconclusive CT pattern } \\
\text { (heterogeneous mass) }\end{array}$ & Metastasis & $27-0.7$ & - \\
\hline
\end{tabular}

Normal values of serum DHEAS $(\mu \mathrm{g} / \mathrm{dl})$ are as follows: men $<40$ years; $70-430$; men $40-60$ years; $60-276$; men $>60$ years; $10-216$; women $<40$ years; $116-505$; women $40-60$ years; $80-422$; women $>60$ years; $21-388$.

DHEAS levels (62\%) included 2 variables, CT diameter of the mass and UFC levels $(r=0.79, P<0.001)$.

The sensitivity, specificity and the predictive values of low DHEAS concentrations for the diagnosis of a benign adrenal mass are given in Table 4 . The table shows the values obtained in the overall series, in the series of incidental masses, and in a series obtained after excluding adrenocortical tumors which were recognizable by other criteria (i.e. catecholamine excess for pheochromocytoma, typical CT picture for myelolipoma, etc.) respectively.

\section{Discussion}

The high rate of discovery of adrenal masses as incidental findings has boosted interest in the differential diagnosis of adrenal lesions. Diagnosis and management of adrenal incidentalomas have great clinical relevance because their discovery could theoretically offer the chance of a presymptomatic detection and treatment of an aggressive disease (4). However, despite the large number of publications devoted to this issue, the diagnostic approach remains a matter of debate.
Algorithms aimed to differentiate benign versus malignant adrenal tumors are often complex and demanding with a consequent large impact on health care costs (4-6).

In the search for a simple and inexpensive tool, we analyzed the diagnostic efficacy of DHEAS measurement to qualify an adrenal mass as benign or malignant. DHEAS levels observed in the patients were categorized as reduced, normal or elevated according to sex- and age-adjusted reference ranges. The working hypothesis was that a low DHEAS level is a marker of benignity. In our series, a low DHEAS level allowed the diagnosis of a benign lesion to be made with a sensitivity of $41 \%$ and a specificity of $100 \%$. Owing to the $100 \%$ specificity, the positive predictive value was also $100 \%$, whereas the negative predictive value and the overall diagnostic accuracy were low. The figure was almost identical when analyzing only the incidental masses and also when restricting the analysis to adrenocortical tumors which were not recognizable by other criteria. In patients with an adrenal carcinoma, DHEAS levels were variable with some overlap with the range of adrenal adenomas. However, only 2 out of 11 adrenal cancers had normal DHEAS levels while in the 
Table 3 Pertinent clinical data and histological diagnoses of the patients with clinically overt adrenal tumors who underwent surgery.

\begin{tabular}{|c|c|c|c|c|c|c|c|}
\hline Patient & Sex & $\begin{array}{c}\text { Age } \\
\text { (years) }\end{array}$ & Clinical presentation & $\begin{array}{l}\text { CT diameter } \\
\text { (cm) }\end{array}$ & Conclusive diagnosis & $\begin{array}{c}\text { DHEAS } \\
(\mu \mathrm{g} / \mathrm{dl})-(\mu \mathrm{mol} / \mathrm{l})\end{array}$ & Stage \\
\hline 1 & M & 60 & Cushing's syndrome & 4.0 & Adrenocortical cancer & $608-16.5$ & IV \\
\hline 2 & $\mathrm{M}$ & 62 & Cushing's syndrome & 8.0 & Adrenocortical cancer & $998-27.0$ & III \\
\hline 3 & $\mathrm{~F}$ & 62 & Abdominal pain & 13.0 & Adrenocortical cancer & $680-18.4$ & IV \\
\hline 4 & $\mathrm{~F}$ & 23 & $\begin{array}{l}\text { Cushing's syndrome, } \\
\text { hypertricosis }\end{array}$ & 7.0 & Adrenocortical cancer & $859-23.3$ & II \\
\hline 5 & $\mathrm{~F}$ & 46 & $\begin{array}{l}\text { Cushing's syndrome, } \\
\text { hypertricosis }\end{array}$ & 8.0 & Adrenocortical cancer & $2000-54.2$ & III \\
\hline 6 & M & 49 & $\begin{array}{l}\text { Abdominal pain, } \\
\text { weight loss }\end{array}$ & 12.0 & Adrenocortical cancer & $105-2.8$ & III \\
\hline 7 & $\mathrm{~F}$ & 46 & Hypertricosis & 12.0 & Adrenocortical cancer & $800-21.7$ & III \\
\hline 8 & $\mathrm{~F}$ & 48 & Hypertricosis & 8.0 & Adrenocortical cancer & $630-17.0$ & III \\
\hline 9 & $\mathrm{~F}$ & 29 & $\begin{array}{l}\text { Abdominal pain, } \\
\text { weight loss }\end{array}$ & 9.0 & Adrenocortical cancer & $290-7.87$ & II \\
\hline 10 & $\mathrm{~F}$ & 33 & Cushing's syndrome & 2.5 & $\begin{array}{l}\text { Cortsol-producing } \\
\text { adrenocortical adenoma }\end{array}$ & $14-0.37$ & - \\
\hline 11 & $\mathrm{~F}$ & 31 & Cushing's syndrome & 3.0 & $\begin{array}{l}\text { Cortisol-producing } \\
\text { adrenocortical adenoma }\end{array}$ & $34-0.9$ & - \\
\hline 12 & $\mathrm{~F}$ & 61 & Cushing's syndrome & 4.0 & $\begin{array}{l}\text { Cortisol-producing } \\
\text { adrenocortical adenoma }\end{array}$ & $137-3.7$ & - \\
\hline 13 & $\mathrm{~F}$ & 34 & Cushing's syndrome & $3.0^{\prime}$ & $\begin{array}{l}\text { Cortisol-producing } \\
\text { adrenocortical adenoma }\end{array}$ & $18-0.48$ & - \\
\hline 14 & $\mathrm{~F}$ & 22 & Cushing's syndrome & 3.8 & $\begin{array}{l}\text { Cortisol-producing } \\
\text { adrenocortical adenoma }\end{array}$ & $20-0.5$ & - \\
\hline 15 & $\mathrm{~F}$ & 15 & Cushing's syndrome & 2.4 & $\begin{array}{l}\text { Cortisol-producing } \\
\text { adrenocortical adenoma }\end{array}$ & $24-0.6$ & - \\
\hline 16 & $\mathrm{~F}$ & 48 & Hypertension & 1.5 & APA & $48-1.3$ & - \\
\hline 17 & M & 49 & $\begin{array}{l}\text { Hypertension, } \\
\text { hypokalemia }\end{array}$ & 2.0 & APA & $195-5.3$ & - \\
\hline 18 & $\mathrm{~F}$ & 24 & $\begin{array}{l}\text { Hypertension, } \\
\text { hypokalemia }\end{array}$ & 3.0 & APA & $19-0.5$ & - \\
\hline 19 & $\mathrm{~F}$ & 36 & Hypertension & 1.0 & APA & $324-8.79$ & - \\
\hline 20 & $\mathrm{M}$ & 45 & Hypertension & 2.0 & APA & $150-4.0$ & - \\
\hline 21 & $\mathrm{~F}$ & 46 & Abdominal pain & 6.5 & Hemorrhagic cyst & $25-0.6$ & - \\
\hline 22 & $\mathrm{~F}$ & 40 & Paroxysmal hypertension & 3.5 & Pheochromocytoma & $95-2.5$ & - \\
\hline 23 & $\mathrm{~F}$ & 50 & Hypertension & 4.0 & Pheochromocytoma & $40-1.0$ & - \\
\hline
\end{tabular}

For normal values of serum DHEAS see legend of Table 2. APA, aldosterone producing adenoma.

remainder DHEAS was elevated or indeed exceedingly high.

Since DHEAS levels are related to demographic variables (16), an accurate definition of the normal range in relation to gender and age is critical. In the present study, different groups of control subjects were employed to produce an accurate matching with the patients in order to eliminate the effect of these

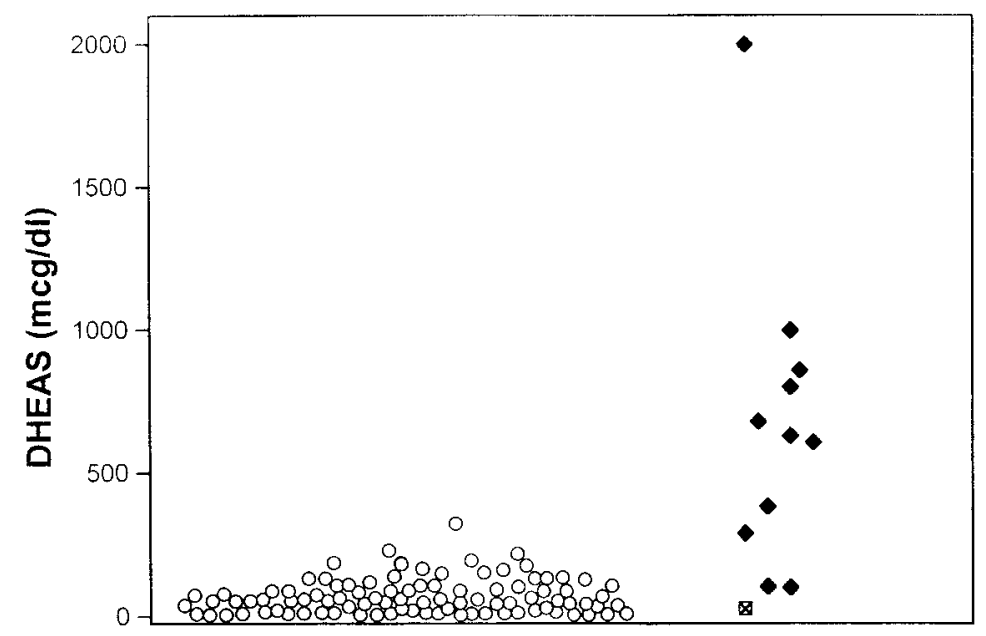

Figure 1 DHEAS levels recorded in patients with benign adrenal masses (open circles) and in patients with adrenal malignancies (primary adrenal cancers, solid diamonds; adrenal metastasis of an extra-adrenal tumor, crossed square). 
Table 4 Diagnostic value of a low DHEAS result as marker of benignity.

\begin{tabular}{|c|c|c|c|c|c|}
\hline & Sensitivity & Specificity & $\begin{array}{l}\text { Positive predictive } \\
\text { value }\end{array}$ & $\begin{array}{l}\text { Negative predictive } \\
\text { value }\end{array}$ & $\begin{array}{l}\text { Diagnostic } \\
\text { accuracy }\end{array}$ \\
\hline $\begin{array}{l}\text { Overall series } \\
\text { (107 cases) }\end{array}$ & $41 \%$ & $100 \%$ & $100 \%$ & $18 \%$ & $47 \%$ \\
\hline $\begin{array}{l}\text { Adrenal incidentalomas } \\
\text { (84 cases) }\end{array}$ & $38 \%$ & $100 \%$ & $100 \%$ & $5 \%$ & $40 \%$ \\
\hline $\begin{array}{l}\text { Adrenal tumors with aspecific features } \\
\text { (85 cases) }\end{array}$ & $41 \%$ & $100 \%$ & $100 \%$ & $12 \%$ & $44 \%$ \\
\hline
\end{tabular}

confounding variables. As a consequence, in a multivariate model, patient age did not significantly contribute to the variance of DHEAS levels.

Some methodological limitations of our study should be addressed, such as its retrospective nature and the lack of pathological diagnosis in every case. However, all presumptive diagnoses of pheochromocytoma or carcinoma were histologically proven, while in the remainder the diagnosis of a benign lesion was based on imaging characteristics of the mass as well as on clinical, endocrine and CT follow-up studies. The combination of these methods, in particular the re-evaluation after an interval of at least 12 months, means that a diagnosis of malignancy, either primary or secondary is very unlikely to be missed $(17,18)$.

It is widely accepted that elevated DHEAS levels have to be considered as suggestive of primary adrenal cancer (11). The significance of high DHEAS as a marker of adrenal cancer has been confirmed also for patients with adrenal incidentalomas in previous work by our group (7). However, other studies have cast doubts on the utility of DHEAS measurement to assess the nature of an adrenal incidentaloma. The works of Flecchia et al. (19), Bencsik et al. (20) and Bernini et al. (21) reported low DHEAS in patients with primary or secondary adrenal malignancies. Different selection criteria could explain the discrepancy with our results since, in these studies, most of the malignant incidentalomas were metastases of extra-adrenal tumors, while only 7 patients with primary adrenal cancers were included. Furthermore, a multicentric study in Italy found that DHEAS measurement was of limited value in the diagnostic approach to an adrenal incidentaloma, but the results of hormone measurement were collected from multiple laboratories with different assay methods employed over a large time span (22).

In the present study, we have looked at DHEAS levels in a different way, testing the hypothesis that low DHEAS may be a marker of benignity rather than analyzing if high DHEAS could predict the presence of a malignancy. The difference is not trivial because a biochemical marker of benignity is more appealing than a marker of malignancy for the clinician dealing with adrenal incidentalomas, as the majority of these masses are actually benign $(5,23)$. The present findings demonstrate that a reduction in DHEAS levels could be useful to assess the benign nature of an adrenal mass in a clinical research setting. If there is local expertise with DHEAS measurement and interpretation of DHEAS results, with special emphasis on the need for a large database of normal subjects to be confident with normal ranges, DHEAS measurement may be of help in the diagnostic approach to an adrenal mass. Since a CT scan is unable to categorize correctly all adrenal masses $(5,23,24)$, the result of DHEAS measurement could complement radiological information in the decision regarding the method of management (surgery vs follow-up), particularly in high surgical risk patients.

However, the value of DHEAS measurement in general practice is limited because it may be difficult to differentiate between low and low-normal values, particularly in the elderly, due to the lack of a threshold value clearly separating adrenal malignancies from benign tumors. Another drawback is that DHEAS levels are found in the normal range in most adrenal tumors and this finding does not allow any diagnostic conclusion. This limitation mainly affects the test sensitivity, which is too low to allow DHEAS measurement to be used as a screening procedure. Moreover, a referral pattern including a number of oncological patients should increase the rate of adrenal metastases discovered incidentally and the present study is not conclusive on the discriminating power of DHEAS in this clinical condition.

\section{References}

1 Glazer HS, Weyman PJ, Sagel SS, Levitt RG \& McClennan BL. Nonfunctioning adrenal masses: incidental discovery on computed tomography. American Journal of Roentgenology 1982139 $81-85$.

2 Mitnick JS, Bosniak MA, Megibow AJ \& Naidich DP. Nonfunctioning adrenal adenomas discovered incidentally on computed tomography. Radiology 198348 495-499.

3 Griffing G. A-I-D-S: the new endocrine epidemic. (Editorial; comment). Journal of Clinical Endocrinology and Metabolism 1994 791530.

4 Chidiac RM \& Aron DC. Incidentalomas: a disease of modern technology. Endocrinology and Metabolism Clinics of North America $199726233-253$.

5 Kloos RT, Gross MD, Francis IR, Korobkin M \& Shapiro B. Incidentally discovered adrenal masses. Endocrine Reviews 1995 $16460-484$.

6 Cook DM \& Loriaux DL. The incidental adrenal mass. American Journal of Medicine 1996101 88-94. 
7 Osella G, Terzolo M, Borretta G, Magro G, Alì A, Piovesan A Paccotti P \& Angeli A. Endocrine evaluation of incidentally discovered adrenal masses (incidentalomas). Journal of Clinical Endocrinology and Metabolism 199479 1532-1539.

8 Ambrosi B, Peverelli S, Passini E, Re T, Ferrario R, Colombo P, Sartorio A \& Faglia G. Abnormalities of endocrine function in patients with clinically 'silent' adrenal masses. European Journal of Endocrinology $1995132422-428$.

9 Barzon L, Scaroni C, Sonino N, Fallo F, Gregianin M, Macrì C \& Boscaro M. Incidentally discovered adrenal tumors: endocrine and scintigraphic correlates. Journal of Clinical Endocrinology and Metabolism 198883 55-62.

10 Fernandez-Real JM, Ricart-Engel W \& Simò R. Pre-clinical Cushing's syndrome: report of three cases and literature review. Hormone Research 199441 230-235.

11 Latronico AC \& Chrousos GP. Extensive personal experience: adrenocortical tumors. Journal of Clinical Endocrinology and Metabolism 199782 1317-1324.

12 Terzolo M, Osella G, Alì A, Borretta G, Cesario F, Paccotti P \& Angeli A. Subclinical Cushing's syndrome in adrenal incidentaloma. Clinical Endocrinology 199848 89-97.

13 Korobkin M, Brodeur FJ, Yutzy GG, Francis IR, Quint LE, Dunnick NR \& Kazerooni EA. Differentiation of adrenal adenomas from nonadenomas using CT attenuation values. American Journal of Roentgenology $1996166531-536$

14 Korobkin M, Brodeur FJ, Francis IR, Quint LE, Dunnick NR \& Goodsitt M. Delayed enhanced CT for differentiation of benign from malignant adrenal masses. Radiology 1996200 737-742.

15 Weiss LM, Medeiros RJ \& Vickery AL. Pathologic features of prognostic significance in adrenocortical carcinoma. American Journal of Surgical Pathology 198913 202-209.

16 Orentreich N, Brind JL, Rizer RL \& Vogelman JH. Age changes and sex differences in serum dehydroepiandrosterone sulfate concentrations throughout adulthood. Journal of Clinical Endocrinology and Metabolism 198459 551-555.
17 Moulton JS. CT of the adrenal glands. Seminars in Roentgenology $198833288-303$.

18 Turner HE, Moore NR, Byrne JV \& Wass JAH. Pituitary, adrenal and thyroid incidentalomas. Endocrine-Related Cancer 19985 131-150.

19 Flecchia D, Mazza E, Carlini M, Blatto A, Olivieri F, Serra G, Camanni F \& Messina M. Reduced serum levels of dehydroepiandrosterone sulphate in adrenal incidentalomas: a marker of adrenocortical tumour. Clinical Endocrinology 199542 129-134.

20 Bencsik Z, Szabolcs I, Kovacs Z, Ferencz A, Voros A, Kaszas I, Bor K, Gonczi J, Goth M, Kovacs L, Dohan O \& Szilagyi G. Low dehydroepiandrosterone sulfate (DHEA-S) level is not a good predictor of hormonal activity in nonselected patients with incidentally detected adrenal tumors. Journal of Clinical Endocrinology and Metabolism 199681 1726-1729.

21 Bernini GP, Argenio GF, Vivaldi MS, Moretti A, Miccoli P, Iacconi P, Magagna A \& Salvetti A. Utility of plasma dehydroepiandrosterone sulphate determination in adrenal incidentalomas. Journal of Endocrinological Investigation 199821 365-371.

22 Mantero F, Masini AM, Opocher G, Giovagnetti M \& Arnaldi G. Adrenal incidentaloma: an overview of hormonal data from the National Italian Study Group. Hormone Research $199747284-$ 289.

23 Terzolo M, Alì A, Osella G \& Mazza E. Prevalence of adrenal carcinoma among incidentally discovered adrenal masses. A retrospective study from 1989 to 1994 . Archives of Surgery 1997 132 914-919.

24 Peppercorn PD, Grossman AB \& Rezneck RH. Imaging of incidentally discovered adrenal masses. Clinical Endocrinology 199848 379-388.

Received 9 August 1999

Accepted 28 February 2000 